\title{
Mechanical Properties and Planar Anisotropy of TC1 Titanium Alloy Sheet
}

\author{
Wu Jianjun, Wang Bao, Liu Weiping
}

Northwestern Polytechnical University, Xi'an 710072, China

\begin{abstract}
This paper investigated the main mechanical properties of TC1 titanium alloy sheets with different thicknesses, and a useful function that can describe the mechanical properties reasonably was used. Moreover, planar anisotropies of above TC1 titanium alloy sheets were studied by Barlat'89 and Yld2000-2d yield functions. The influence of $M$ value was analyzed on describing ability for distribution characteristics of material yield stress and anisotropic coefficient, and their performances were compared on the titanium alloy sheets.
\end{abstract}

Key words: TC1; mechanical properties; yield function

Titanium and its alloys are widely used in aerospace, biomedical, defense and energy industry due to their high strength-mass ratio, corrosion resistance, biocompatibility and heat resistance ${ }^{[1]}$. Particularly, in the application of aeronautics and astronautics, mass reduction can enhance aircraft performances, and reduce manufacturing cost ${ }^{[2]}$.

Owing to the inherent crystallographic texture and rolling process, TC1 titanium alloy sheet exhibits strong planar plastic anisotropy ${ }^{[3]}$. Therefore, research on the mechanical properties in a certain direction is not enough to describe the material properties clearly.

When studying the mechanical properties, especially for strong plastic anisotropy, many anisotropic yield functions such as Hill (1948), Hill (1979), Barlat and Lian (1989), Barlat et al. (2003), Plunkett et el. $(2008)^{[4-8]}$ have been developed so far.

One objective of the paper is to select a function model which can predict the mechanical properties of the TC1 titanium alloy in any direction reasonably. Another one is to evaluate the performance of two widely used anisotropic yield functions, Barlat'89 and Yld2000-2d, in describing the plastic planar anisotropy of the TC1 titanium alloy sheets.

The exponent $M$ in Barlat'89/Yld2000-2d yield function is related to crystallographic structure of the material, whose value is 8 for face-centered cubic (fcc) materials and 6 for body-centered cubic (bcc) materials. For (hexagonal close packed) HCP metal, such as TC1, the value of $M$ is unknown. In the present paper, a reasonable value of exponent $M$, which can describe plastic anisotropy more accurately, will be discussed by calculation, analysis, and experimental data.

\section{Experiment}

Up to now, extensive experimental observations have demonstrated that the mechanical properties of Ti titanium alloy sheet represent a strong plastic anisotropy ${ }^{[9]}$. In this paper, TC1 titanium alloy sheet with three different thicknesses were selected, and its components are listed in Table 1.

The dimensions of the specimen for tensile tests are shown in Fig.1. Specimens with thickness 0.6 and $0.8 \mathrm{~mm}$ were extracted at 5 different angles with intervals of $22.5^{\circ}$, $0^{\circ}$ (rolling direction), $22.5^{\circ}, 45^{\circ}$ (diagonal direction), $67.5^{\circ}$ and $90^{\circ}$ (transverse direction).

A universal testing machine was used to measure the yield stress $\sigma_{0.2}$, the ultimate tensile strength $\sigma_{\mathrm{b}}$, the uniform tensile strain $\varepsilon_{\mathrm{u}}$, the percentage area reduction $\Phi_{\mathrm{f}}$, and the anisotropic index $r_{\text {pmax }}\left(r=\varepsilon_{b} / \varepsilon_{t}\right)$ under the maximum

$\overline{\text { Received date: March 25, } 2016}$

Foundation item: National Natural Science Foundation of China (51075332)

Corresponding author: Wu Jianjun, Ph. D., Professor, School of Mechanical Engineering, Northwestern Polytechnical University, Xi'an 710072, P. R. China, Tel 0086-29-88493101, E-mail: wujj@nwpu.edu.cn

Copyright (C) 2017, Northwest Institute for Nonferrous Metal Research. Published by Elsevier BV. All rights reserved. 


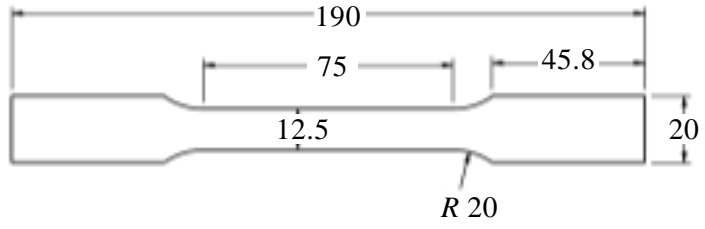

Fig.1 Schematic of the specimen geometry

load. Table 2 Table 4 list material properties obtained by above experiments.

Based on Table 2, for the TC1 titanium alloy sheet of 0.6 $\mathrm{mm}$ and $0.8 \mathrm{~mm}$ thickness, the highest value of $\sigma_{\mathrm{b}}$ is in the rolling direction, the second in the transverse direction, and the lowest in the diagonal direction. According to the difference between the rolling and transverse direction, the lowest value should exist in a direction, which is greater than $45^{\circ}$.

However, there is a different result for the $2.0 \mathrm{~mm}$ thickness. The value of $\sigma_{\mathrm{b}}$ along the transverse direction is the highest, the second is in the rolling, and the lowest is in the diagonal direction.

For the value of $\varepsilon_{\mathrm{u}}$, the range of the present paper is bigger than other experimental material values, and the reason may be the influence of the experimental error.

In Table 4, one can draw a conclusion that for the TC1 titanium alloy sheet of 0.6 and $0.8 \mathrm{~mm}$ in thickness, the value of $\Phi_{\mathrm{f}}$ increases as the angle between the tensile direction and rolling direction increases. For the thickness of $2.0 \mathrm{~mm}$, the value of $\Phi_{\mathrm{f}}$ along the rolling direction is the smallest.

Table 1 Chemical composition of TC1 alloy sheet (wt\%)

\begin{tabular}{|c|c|c|c|c|c|c|c|c|c|}
\hline Thickness/mm & $\mathrm{C}$ & $\mathrm{Mn}$ & $\mathrm{Si}$ & $\mathrm{Fe}$ & $\mathrm{Al}$ & $\mathrm{N}$ & $\mathrm{H}$ & $\mathrm{O}$ & $\mathrm{Ti}$ \\
\hline 0.6 & 0.09 & 1.54 & 0.09 & 0.055 & 1.75 & 0.025 & 0.0084 & 0.12 & Balance \\
\hline 0.8 & $<0.047$ & 1.51 & 0.10 & 0.03 & 1.66 & 0.017 & 0.0044 & 0.103 & Balance \\
\hline 2.0 & 0.07 & 1.37 & 0.03 & 0.07 & 1.43 & 0.027 & 0.0105 & & Balance \\
\hline
\end{tabular}

Table 2 Values of $\sigma_{b}$ in uniaxial experiments

\begin{tabular}{cccc}
\hline Loading dir. & $\begin{array}{c}\text { Thickness } \\
\text { /mm }\end{array}$ & $\begin{array}{c}\text { Range } \\
/ \mathrm{MPa}\end{array}$ & $\begin{array}{c}\text { Average } \\
\text { /MPa }\end{array}$ \\
\hline Rolling direction & 0.6 & $729 \sim 733$ & 731 \\
Diagonal direction & 0.6 & $696 \sim 700$ & 697 \\
Transverse direction & 0.6 & $711 \sim 718$ & 714 \\
Rolling direction & 0.8 & 710 & 710 \\
Diagonal direction & 0.8 & $672 \sim 680$ & 675 \\
Transverse direction & 0.8 & $685 \sim 687$ & 686 \\
Rolling direction & 2.0 & $823 \sim 827$ & 825 \\
Diagonal direction & 2.0 & $795 \sim 805$ & 800 \\
Transverse direction & 2.0 & $854 \sim 871$ & 863 \\
\hline
\end{tabular}

Table 3 Values of $\varepsilon_{\mathrm{u}}$ in uniaxial experiments

\begin{tabular}{cccc}
\hline Loading dir. & $\begin{array}{c}\text { Thickness } \\
/ \mathrm{mm}\end{array}$ & $\begin{array}{c}\text { Range } \\
/ \%\end{array}$ & $\begin{array}{c}\text { Average } \\
/ \%\end{array}$ \\
\hline Rolling direction & 0.6 & $10.8 \sim 11.8$ & 11.3 \\
Diagonal direction & 0.6 & $8.8 \sim 10.0$ & 9.3 \\
Transverse direction & 0.6 & $7.3 \sim 9.4$ & 8.1 \\
Rolling direction & 0.8 & $13.0 \sim 14.0$ & 13.5 \\
Diagonal direction & 0.8 & $9.8 \sim 10.3$ & 10.07 \\
Transverse direction & 0.8 & $7.2 \sim 7.6$ & 7.4 \\
Rolling direction & 2.0 & $9.3 \sim 9.4$ & 9.3 \\
Diagonal direction & 2.0 & $5.0 \sim 7.3$ & 5.8 \\
Transverse direction & 2.0 & 4.0 & 4.0 \\
\hline
\end{tabular}

Table 4 Values of $\Phi_{\mathrm{f}}$ in uniaxial experiments

\begin{tabular}{cccc}
\hline Loading dir. & $\begin{array}{c}\text { Thickness } \\
/ \mathrm{mm}\end{array}$ & $\begin{array}{c}\text { Range } \\
/ \%\end{array}$ & $\begin{array}{c}\text { Average } \\
/ \%\end{array}$ \\
\hline Rolling direction & 0.6 & $43.9 \sim 44.8$ & 44.2 \\
Diagonal direction & 0.6 & $47.2 \sim 49.1$ & 48.3 \\
Transverse direction & 0.6 & $51.9 \sim 53.4$ & 52.4 \\
Rolling direction & 0.8 & $43.6 \sim 45.3$ & 44.2 \\
Diagonal direction & 0.8 & $47.47 \sim 47.54$ & 47.5 \\
Transverse direction & 0.8 & $51.0 \sim 53.0$ & 52.0 \\
Rolling direction & 2.0 & $37.5 \sim 37.9$ & 37.7 \\
Diagonal direction & 2.0 & 50.3 & 50.3 \\
Transverse direction & 2.0 & $42.9 \sim 48.0$ & 45.6 \\
\hline
\end{tabular}

\section{A Useful Function Model}

On the above analysis related to the material properties along three directions, a useful function model was used to describe the mechanical properties in any direction.

According to experimental phenomenon of the deep drawing for TC1 cylindrical parts, the deformation has a symmetric feature in the rolling and transverse directions. In other words, the mechanical properties along the rolling direction and the transverse direction have extreme values. Meanwhile, for the anisotropy materials, it's enough to represent material properties of any direction just in one quadrant. Therefore, the distribution of the mechanical properties should be a function of $\cos 2 n \theta$, where $\theta$ is the angle between a certain direction and the rolling direction, and $n$ is a positive integer.

In addition, as mentioned before, many kinds of properties have a maximum or minimum value in a certain direction that is larger or smaller than $45^{\circ}$. It means that the mechanical property has another extreme value between $0^{\circ} \sim 90^{\circ}$

Consequently, the easiest function, describing the distribution of mechanical properties, should be expressed as follows:

$$
A=A_{c p}+A_{2} \cos 2 \theta+A_{4} \cos 4 \theta
$$

Where $A$ is some kind of mechanical property, such as $\sigma_{\mathrm{b}}, \sigma_{0.2}$, $\Phi_{\mathrm{f}} . A_{\mathrm{cp}}$ is the average value of this property. $A_{2}, A_{4}$ are two coefficients calculated by the experimental data in the rolling, transverse and diagonal directions. Taking a derivative of Eq. (1) with respect to $\theta$, the result is shown in Eq.(2): 


$$
\mathrm{d} A / \mathrm{d} \theta=-2 A_{2} \sin 2 \theta-4 A_{4} \sin 4 \theta=-2\left(A_{2}+4 A_{4} \cos 2 \theta\right) \sin 2 \theta
$$

We can get $\mathrm{d} A / \mathrm{d} \theta=0$, when $\theta=0, \pi / 2, \pi, 3 \pi / 2,2 \pi$. This satisfies the condition that the mechanical properties have extreme values in rolling and transverse directions. Furthermore, if $\alpha$ also satisfies the above condition, we obtain Eq.(3)

$$
\left.\cos 2 \theta\right|_{\theta=\alpha}=\cos 2 \alpha=-A_{2} / 4 A_{4}
$$

Where $\alpha$ represents a direction between $0^{\circ} \sim 90^{\circ}$ and $\mathrm{d} A / \mathrm{d} \theta$ is equal to 0 . Therefore, as long as $A_{4} \neq 0$, there must exists another extreme point in a direction between $0^{\circ}$ to $90^{\circ}$. The maximum or minimum value is shown in Eq.(4):

$$
A_{\max } \text { or } A_{\min }=A_{\mathrm{cp}}-A_{4}\left(1+2 \cos ^{2} 2 \alpha\right)
$$

The $A_{\mathrm{cp}}, A_{2}$ and $A_{4}$ are given by Eq.(5):

$$
\begin{aligned}
& A_{\mathrm{cp}}=\left(A_{0^{\circ}}+2 A_{45^{\circ}}+A_{90^{\circ}}\right) / 4 \\
& A_{2}=\left(A_{0^{\circ}}-A_{90^{\circ}}\right) / 2 \\
& A_{4}=\left(A_{0^{\circ}}+A_{90^{\circ}}-2 A_{45^{\circ}}\right) / 4
\end{aligned}
$$

In this paper, $A_{0^{\circ}}, A_{45^{\circ}}$ and $A_{90^{\circ}}$ represent the mechanical properties in three different directions, respectively.

The effectiveness of the trigonometric function mentioned above has been demonstrated in related Ref.[9].

\section{Performance of Two Yield Functions}

All parameters of these yield functions, Barlat' 89 and Yld2000-2d, are obtained from the $r$ values and the values of yield stress in three directions. These values with three different thicknesses are listed in Table 5 Table 7.

\subsection{Fundamental review on anisotropic yield functions}

For describing the plastic anisotropy of TC1 titanium alloy sheet, it is essential to select a suitable yield criterion. Two widely used anisotropic yield functions, Barlat'89 and Yld2000-2d, were chosen to investigate the planar anisotropy of TC1. The first yield function needs a small number of experimental data required for calibration. The second one requires more material data to identify the anisotropy coefficients and thus it is more flexible than the first one.

Barlat et al. have made a significant contribution to anisotropic yield criteria such as Barlat'89 and Yld2000-2d. Because its expression format is simple and its anisotropy parameter can be obtained easily, Barlat' 89 yield criterion is used widely for anisotropic material in numerical simulation. In plane stress condition, Barlat' 89 is expressed as in Eq.(6)

$$
f=a\left|K_{1}+K_{2}\right|^{M}+a\left|K_{1}-K_{2}\right|^{M}+c\left|2 K_{2}\right|^{M}=2 \bar{\sigma}^{M}
$$

with

$$
K_{2}=\sqrt{\left(\frac{\sigma_{x x}-h \sigma_{y y}}{2}\right)^{2}+p^{2} \sigma_{x y}^{2}} \quad K_{1}=\frac{\sigma_{x x}+h \sigma_{y y}}{2}
$$

Where $a, c, h$, and $p$ are material constants. The exponent $M$ is typically chosen based on the crystal plasticity calculations of Logan and Hosford ${ }^{[10]}$. Following their recommendation for crystallographic structure material, the values of $M$ is 8 for body-centered cubic (bcc) and 6 for face-centered cubic (fcc) materials ${ }^{[11]}$. For hcp metal, such as TC1, the value of $M$ is unknown. It is recommended for $8 \sim 12$ by Guo et al ${ }^{[12]}$.

Yld2000-2d yield function is expressed as in Eq.(7), where recommended values of the exponent $M$ is 8 for face-centered cubic (fcc) materials and 6 for body -centered cubic (bcc) materials.

$$
\phi=\left|X_{1}^{\prime}-X_{2}^{\prime}\right|^{M}+\left|2 X_{2}^{\prime \prime}+X_{1}^{\prime \prime}\right|^{M}+\left|2 X_{1}^{\prime \prime}+X_{2}^{\prime \prime}\right|^{M}=2 \bar{\sigma}^{M}
$$

where, $X_{j}^{\prime \prime}$ and $X_{k}^{\prime \prime}$ are the principle values of $X^{\prime}$ and $X^{\prime \prime}$ defined as

$$
\begin{aligned}
& X^{\prime}=C^{\prime} s=C^{\prime} T \sigma=L^{\prime} \sigma \\
& X^{\prime \prime}=C^{\prime \prime} s=C^{\prime \prime} T \sigma=L^{\prime \prime} \sigma
\end{aligned}
$$

With

\begin{tabular}{|c|c|c|c|c|c|c|}
\hline & $r_{0}$ & $r_{45}$ & $r_{90}$ & $\sigma_{0} / \mathrm{MPa}$ & $\sigma_{45} / \mathrm{MPa}$ & $\sigma_{90} / \mathrm{MPa}$ \\
\hline Numerical range & $0.73 \sim 0.78$ & $2.05 \sim 2.1$ & $2.0 \sim 2.05$ & $625 \sim 644$ & $650 \sim 676$ & $677 \sim 685$ \\
\hline Average & 0.75 & 2.1 & 2.05 & 635 & 663 & 681 \\
\hline
\end{tabular}

$$
\left[\begin{array}{l}
L_{11}^{\prime} \\
L_{12}^{\prime} \\
L_{21}^{\prime} \\
L_{22}^{\prime} \\
L_{66}^{\prime}
\end{array}\right]=\left[\begin{array}{ccc}
2 / 3 & 0 & 0 \\
-1 / 3 & 0 & 0 \\
0 & -1 / 3 & 0 \\
0 & 2 / 3 & 0 \\
0 & 0 & 1
\end{array}\right]\left[\begin{array}{l}
\alpha_{1} \\
\alpha_{2} \\
\alpha_{7}
\end{array}\right]\left[\begin{array}{l}
L_{11}^{\prime \prime} \\
L_{12}^{\prime \prime} \\
L_{21}^{\prime \prime} \\
L_{22}^{\prime \prime} \\
L_{66}^{\prime \prime}
\end{array}\right]=\frac{1}{9}\left[\begin{array}{ccccc}
-2 & 2 & 8 & -2 & 0 \\
1 & -4 & -4 & 4 & 0 \\
4 & -4 & -4 & 1 & 0 \\
-2 & 8 & 2 & -2 & 0 \\
0 & 0 & 0 & 0 & 9
\end{array}\right]\left[\begin{array}{l}
\alpha_{3} \\
\alpha_{4} \\
\alpha_{5} \\
\alpha_{6} \\
\alpha_{8}
\end{array}\right](7 \mathrm{~b})
$$

This yield criterion is isotropic if the anisotropy coefficient $\alpha_{i}$ (for $i$ from 1 to 8 ) decreases to 1. Uniaxial tension tests along the rolling, diagonal and transversal direction provide six input data points $\left(\sigma_{0}, \sigma_{45}, \sigma_{90}, r_{0}\right.$

Table 5 Experimental values of material parameters for TC1 titanium alloy sheet of $0.6 \mathrm{~mm}$ thickness

\begin{tabular}{ccccccc}
\hline & $r_{0}$ & $r_{45}$ & $r_{90}$ & $\sigma_{0} / \mathrm{MPa}$ & $\sigma_{45} / \mathrm{MPa}$ & $\sigma_{90} / \mathrm{MPa}$ \\
\hline Numerical range & $2.05 \sim 1.37$ & $2.95 \sim 3.56$ & $3.00 \sim 3.20$ & $512 \sim 514$ & $507 \sim 507.5$ & $562 \sim 588$ \\
Average & 2.21 & 3.26 & 3.10 & 513 & 507 & 575 \\
\hline
\end{tabular}

Table 6 Experimental values of material parameters for TC1 titanium alloy sheet of $0.8 \mathrm{~mm}$ thickness

\begin{tabular}{ccccccc}
\hline & $r_{0}$ & $r_{45}$ & $r_{90}$ & $\sigma_{0} / \mathrm{MPa}$ & $\sigma_{45} / \mathrm{MPa}$ & $\sigma_{90} / \mathrm{MPa}$ \\
\hline Numerical range & $2.05 \sim 2.24$ & $3.96 \sim 4.07$ & $3.20 \sim 3.28$ & $553 \sim 565$ & $531 \sim 538$ & 555 \\
Average & 2.16 & 4.0 & 3.24 & 559 & 534 & 555 \\
\hline
\end{tabular}

Table 7 Experimental values of material parameters for TC1 titanium alloy sheet of $2.0 \mathrm{~mm}$ thickness 
Table 8 Anisotropic coefficients for Barlat'89 yield function

\begin{tabular}{ccccc}
\hline Thickness/mm & $a$ & $h$ & $p(M=8)$ & $p(M=10)$ \\
0.6 & 0.5570 & 0.9542 & 1.0264 & 1.0282 \\
2.8 & 0.5546 & 0.9458 & 1.0635 & 1.0668 \\
\hline
\end{tabular}
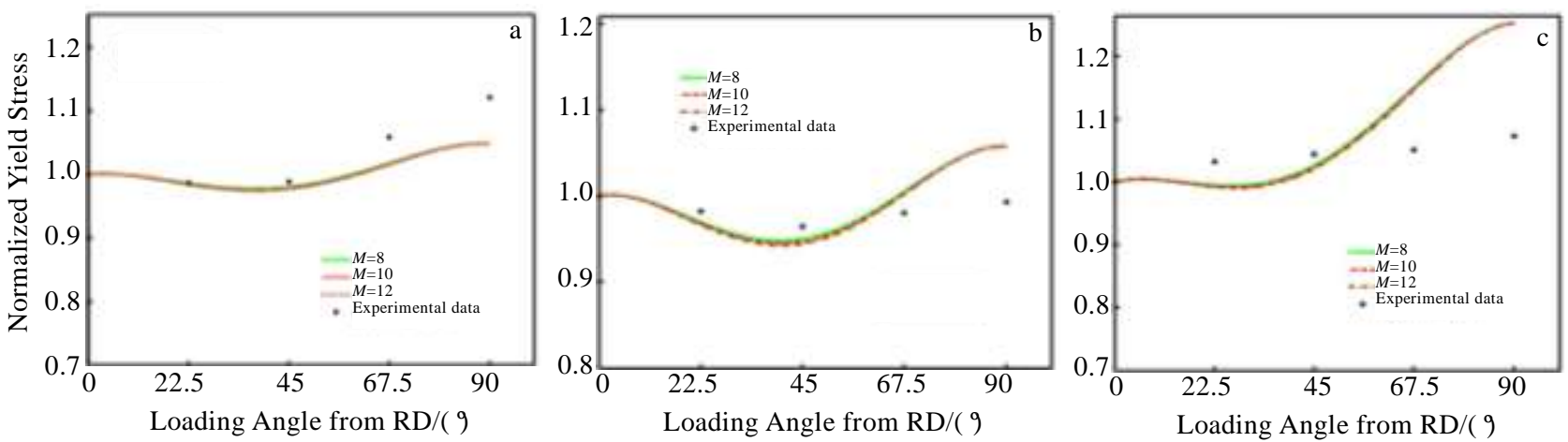

Fig.2 Comparison of experimental and predicted yield stress distribution: (a) $0.6 \mathrm{~mm}$, (b) $0.8 \mathrm{~mm}$, and (c) $2.0 \mathrm{~mm}$
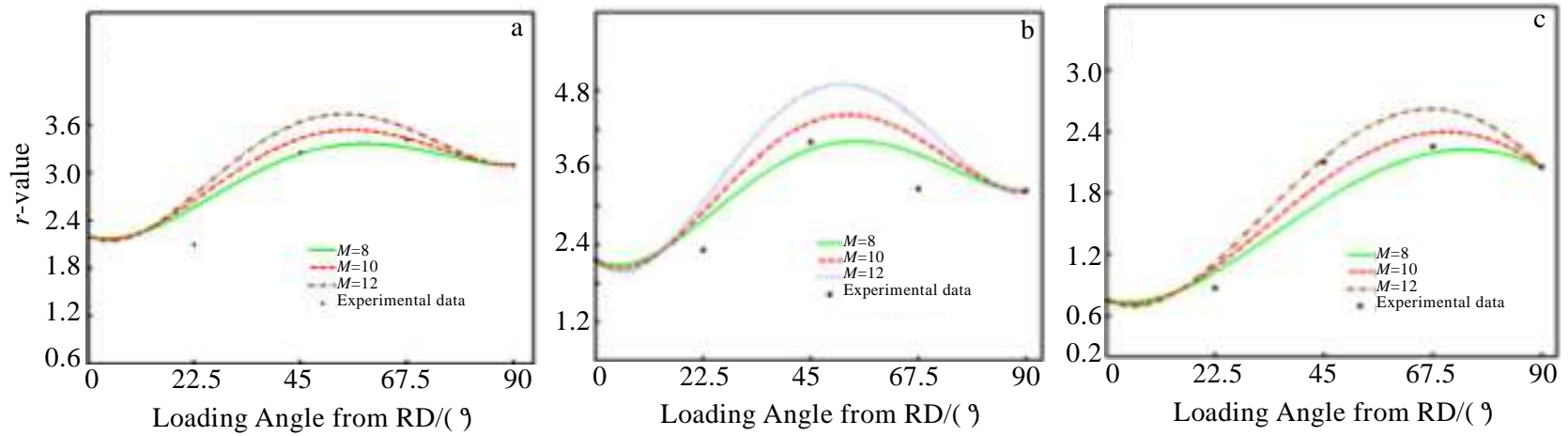

Fig.3 Comparison of experimental and predicted $r$ values distribution: (a) $0.6 \mathrm{~mm}$, (b) $0.8 \mathrm{~mm}$, and (c) $2.0 \mathrm{~mm}$

$r_{45}$ and $\left.r_{90}\right)$ to determine the coefficients of the Yld2000$2 \mathrm{~d}$ yield function. The ratio of the bi-axial yield stress to yield stress in rolling direction, $\sigma_{\mathrm{b}} / \sigma_{0}$, is additionally required to calibrate the Yld2000-2d yield function ${ }^{[7]}$. However, in this paper, considering the limitation of experimental condition, the value of $\sigma_{\mathrm{b}}$ was estimated on the average of $\sigma_{0}$ and $\sigma_{90}$. In this way, only seven coefficients are needed to account for the seven input data mentioned above. The eighth coefficient was identified using the practical assumption, $L_{12}^{\prime \prime}=L_{21}^{\prime \prime}$ following Yoon et $\mathrm{al}^{[13]}$.

\subsection{Evaluation of different exponent $M$ in Barlat'89 yield function}

For Barlat' 89 yield function, the parameter $p$ varies with the change of $M$. As the method mentioned in Section 3.1, using $r$ values along $0^{\circ}, 45^{\circ}, 90^{\circ}$, the material coefficients for TC1 sheet sample are listed in Table 8 .

Fig.2 shows the predictions of the yield stress distribution. No matter which value of $M$ is chosen, their performances to describe the plastic anisotropy of the target materials are the same. The comparisons of the $r$ value distribution are plotted in Fig. 3 for $0.6 \mathrm{~mm}, 0.8 \mathrm{~mm}$ and 2.0 $\mathrm{mm}$. It is noted that the Barlat' 89 yield model with $M=8$ shows better agreements with experiments than the other two exponents.

In conclusion, the result from Barlat' 89 criterion with $M=8$ exhibits much better agreement with experiments than other two $M$ values. But the $r$ values and the yield stress distribution can't be more accurately described. Therefore, Yld2000-2d yield function also needs to be chosen to evaluate its performance to describe the plastic anisotropy of this material.

\subsection{Evaluation of different exponent $M$ in Yld2000. 2d yield function}

As mentioned in Section 3.1. Yld2000-2d yield function requires more material data to identify the anisotropy coefficients.

The same problem occurs in Yld2000-2d yield function, because the parameter $\alpha_{i}$ is varied with the change of $M$, which affects the predicted effect. The anisotropy 
coefficients of the Yld2000-2d yield criterion are tabulated at various exponents $M$ in Tables 9 11 for thickness $0.6,0.8$ and $2.0 \mathrm{~mm}$.

As shown in Fig.4 and Fig.5, the value of $M$ does not much influence the performance to describe the plastic anisotropy of the target materials. However, it should be noted that the predicted yield stress distribution is less accurate than the other two when $M=12$. So, in the following comparison among different yield criterions, the value of $M$ can be determined to be 8 .

\section{Evaluation of Anisotropic Yield Criteria}

Table 9 Anisotropic coefficients for YLD2000-2D yield function when exponent $M$ is 8

\begin{tabular}{cccccccccc}
\hline Thickness/mm & $\alpha_{1}$ & $\alpha_{2}$ & $\alpha_{3}$ & $\alpha_{4}$ & $\alpha_{5}$ & $\alpha_{6}$ & $\alpha_{7}$ \\
\hline 0.6 & 1.1916 & 0.7983 & 1.0362 & 0.8882 & 0.9199 & 1.0045 & 1.0673 & 0.8855 \\
0.8 & 1.0109 & 1.0991 & 1.1039 & 0.9458 & 0.9761 & 1.0736 & 1.1029 & 0.8244 \\
2.0 & 0.9756 & 0.9701 & 1.0232 & 0.9129 & 1.0006 & 0.9356 & 0.9988 & 0.8330 \\
\hline
\end{tabular}

Table 10 Anisotropic coefficients for YLD2000-2D yield function when exponent $M$ is 10

\begin{tabular}{ccccccccc}
\hline Thickness/mm & $\alpha_{1}$ & $\alpha_{2}$ & $\alpha_{3}$ & $\alpha_{4}$ & $\alpha_{5}$ & $\alpha_{6}$ & $\alpha_{7}$ \\
\hline 0.6 & 1.1719 & 0.7967 & 1.0225 & 0.8928 & 0.9331 & 0.9822 & 1.0550 & 0.9276 \\
0.8 & 1.0083 & 1.0794 & 1.0807 & 0.9596 & 0.9809 & 1.0593 & 1.0887 & 0.8766 \\
2.0 & 1.0012 & 0.9417 & 1.0119 & 0.9219 & 0.9963 & 0.9375 & 0.9988 & 0.8586 \\
\hline
\end{tabular}

Table 11 Anisotropic coefficients for YLD2000-2D yield function when exponent $M$ is 12

\begin{tabular}{cccccccccc}
\hline Thickness/mm & $\alpha_{1}$ & $\alpha_{2}$ & $\alpha_{3}$ & $\alpha_{4}$ & $\alpha_{5}$ & $\alpha_{6}$ & $\alpha_{7}$ \\
\hline 0.6 & 1.1598 & 0.7951 & 1.0136 & 0.8959 & 0.9413 & 0.9682 & 1.0470 & 0.9549 \\
0.8 & 1.0062 & 1.0671 & 1.0662 & 0.9682 & 0.9843 & 1.0501 & 1.0796 & 0.9105 & 0.952 \\
2.0 & 1.0157 & 0.9254 & 1.0057 & 0.9269 & 0.9940 & 0.9386 & 0.9840 & 0.8749 \\
\hline
\end{tabular}
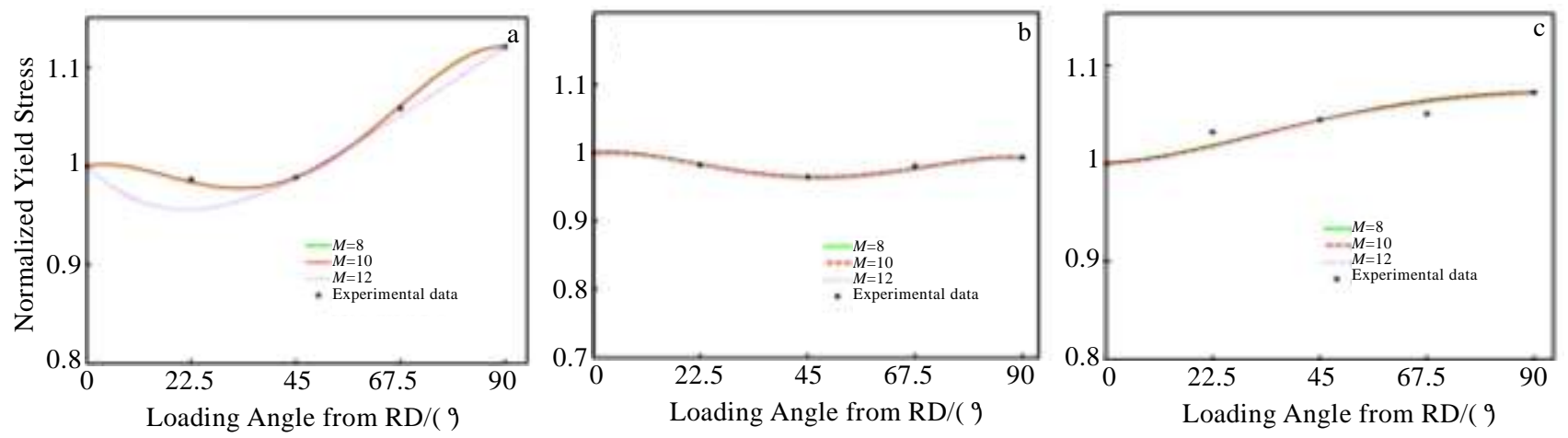

Fig.4 Comparison of experimental and predicted yield stress distribution: (a) $0.6 \mathrm{~mm}$, (b) $0.8 \mathrm{~mm}$, and (c) $2.0 \mathrm{~mm}$
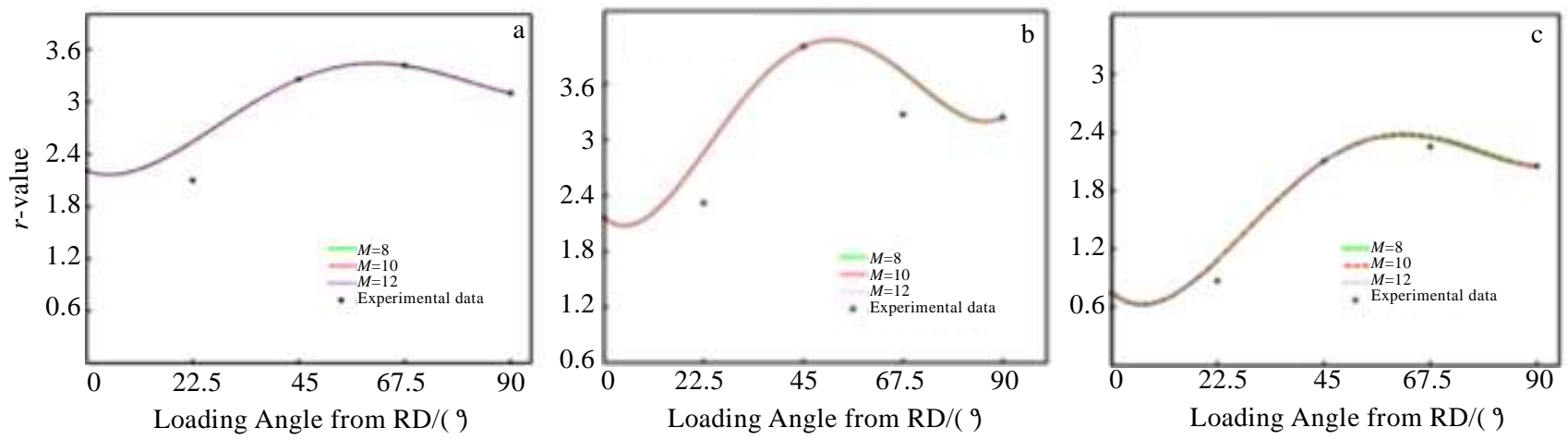

Fig.5 Comparison of experimental and predicted $r$ values distribution: (a) $0.6 \mathrm{~mm}$, (b) $0.8 \mathrm{~mm}$, and (c) $2.0 \mathrm{~mm}$ 
results at $2.0 \mathrm{~mm}$ thickness are not so coincident. For the sheet of $0.8 \mathrm{~mm}$ thickness, the theoretically calculated planar distributions of the $r$ value have a greater deviation with the experimental data. It should be noted that the trigonometric function is simple and easy to predict any mechanical properties. What is more, as shown in Fig. 6, the precision of trigonometric function has a very small difference with Barlat'89 yield criterion for predicting $r$ value.

The comparisons of yield stress are illustrated in Fig.7. The distribution calculated with Yld2000-2d, however, displays a strong consistency with the experimental yield stress distribution.
Concerning the TC1 titanium alloy sheet of $0.6 \mathrm{~mm}$ and $0.8 \mathrm{~mm}$ in thickness, the distribution calculated with Yld2000-2d-2d and trigonometric function displays a strong consistency with the experimental yield stress distribution. About the TC1 titanium alloy sheet of $2.0 \mathrm{~mm}$, results from all yield criteria differ from the experimental value except the Yld2000-2d yield criteria.

This clarifies that the yield criterion based on Yld2000-2d can better describe the plastic anisotropy of the TC1 titanium alloy sheet than other methods despite of its complicated formulation. Therefore, Yld2000-2d yield criterion will be the best method to describe the anisotropic yield locus until now.
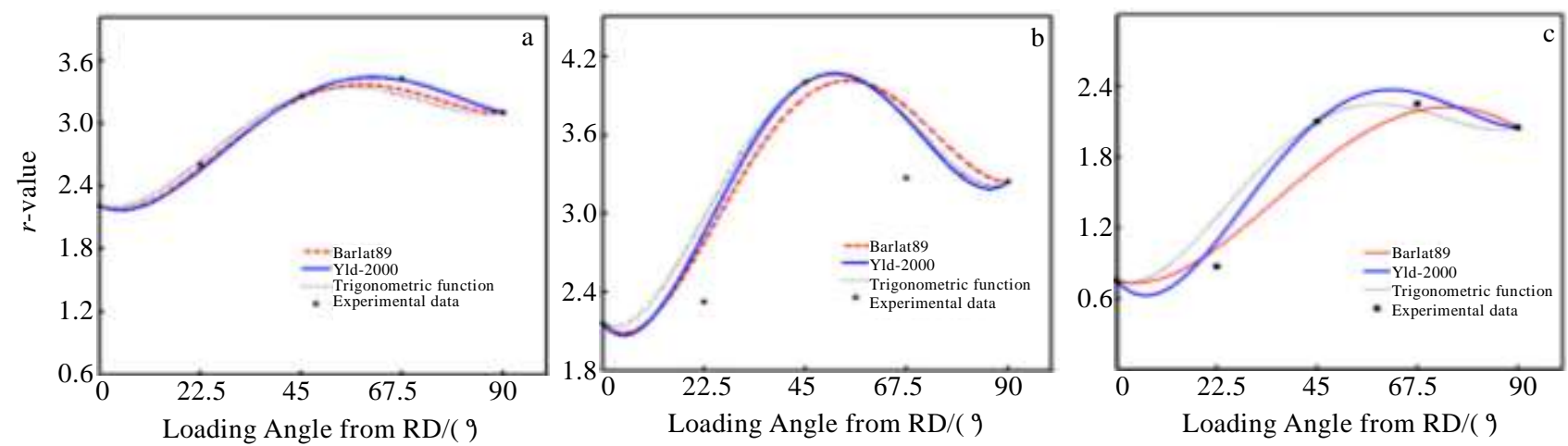

Fig.6 Comparisons of the $r$-value: (a) $0.6 \mathrm{~mm}$, (b) $0.8 \mathrm{~mm}$, and (c) $2.0 \mathrm{~mm}$
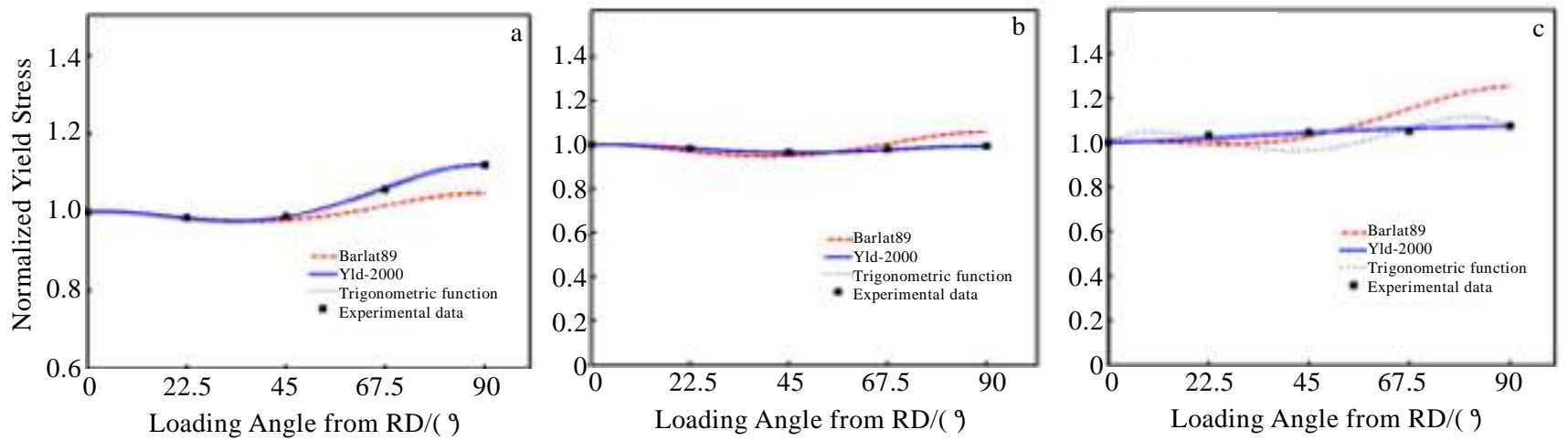

Fig.7 Comparisons of the yield stress distribution: (a) $0.6 \mathrm{~mm}$, (b) $0.8 \mathrm{~mm}$, and (c) $2.0 \mathrm{~mm}$

\section{Conclusions}

1) A trigonometric function can be used to predict mechanical properties in any direction reasonably.

2) For TC1 titanium alloy sheet, Barlat' 89 yield model with $M=8$ shows better agreements with experiments than the other two exponents. For Yld2000-2d yield model, no matter which value of $M$ is chosen, the performances of $M$ to describe the plastic anisotropy of the target materials are the same.

3) Yld2000-2d yield function can be used to accurately describe the plastic anisotropy of the TC1 titanium alloy sheet.

\section{References}

1 Nixon M E, Cazacu O, Lebensohn R A. International Journal of Plasticity[J], 2010, 26: 516

2 Kosaka Y, Stephen P, Faller K. JOM[J], 2004, 56(11): 32

3 Zhu Z S, Liu R Y, Yan M G et al. J Mater Sci[J], 1997, 32: 5163

4 Hill R. Proceedings of the Royal Society of London Seres A Mathematical and Physical Science [J], 1948, 193(1033): 281

5 Hill R J. Physics and Chemistry of Minerals[J], 1979, 5: 179

6 Barlat F, Lian K. International Journal of Plasticity[J], 1989, 5(1): 51

7 Barlat F, Brem J C, Yoon J W et al. International Journal of 
Plasticity[J], 2003, 19: 1297

8 Plunkett B, Cazacu O, Barlat F. International Journal of Plasticity[J], 2008, 24: 847

9 Wu Jianjun, Zhou Weixian. Basic Theory of Sheets Formability[M]. Xi'an: Northwestern Polytechnical University Press, 2010

10 Logan R W, Hosford W F. Int J Mech Sci[J], 1980, 22: 419
11 Hosford W F. The Mechanics of Crystals and Textured Polycrystals[M]. New York: Oxford University Press, 1993: 201

12 Guo W, Zhou F, Chen J W. J Jilin Univ Tecnol[J], 1993, 23(1): 36

13 Yoon J, Barlat F, Dick R E et al. International Journal of Plasticity[J], 2004, 20: 495

\title{
TC1 钛合金板的机械性能和面内各向异性
}

\author{
吴建军, 王 苍, 刘伟萍 \\ (西北工业大学, 陕西 西安 710072)
}

\begin{abstract}
摘 要: 首先全面研究了不同厚度TC1钛合金板料在不同方向的机械性能。在此基础上，介绍了一种能够有效描述不同厚度下的 TC1钛 合金板料机械性能分布规律的三角函数。此外，重点分析研究了Barlat'89和Yld $2000-2 \mathrm{~d}$ 屈服准则中指数 $M$ 对材料屈服应力和厚向异性指 数分布规律描述能力的影响, 并比较了上述 2 种屈服准则对不同厚度 $\mathrm{TC} 1$ 钛合金板料的适应性。
\end{abstract}

关键词: $\mathrm{TC} 1$; 机械性能; 函数模型

作者简介: 吴建军, 男, 1963 年生, 博士, 教授, 西北工业大学机电学院, 陕西 西安 710072, 电话: 029-88493101, E-mail: wujj@nwpu.edu.cn 